\title{
THE BUNDLE PLOT: EVOLUTION OF SYMBOLIC SPACE UNDER THE SYSTEM PARAMETER CHANGES
}

\author{
JIONGXUAN ZHENG*, JOSEPH D. SKUFCA ${ }^{\dagger}$ \\ and ERIK M. BOLLT ${ }^{\ddagger}$ \\ Mathematics and Computer Science, \\ Clarkson University, Potsdam, \\ NY 13699, United States \\ *jizheng@clarkson.edu \\ †jskufca@clarkson.edu \\ †bolltem@clarkson.edu
}

Received November 29, 2012

\begin{abstract}
This paper provides a topological dynamics perspective on the full bifurcation unfolding in unimodal mappings. We present a bundle structure, visualized as a bundle plot, to show the evolution of symbolic space as we vary a system parameter. The bundle plot can be viewed as a limit process of an assignment plot, which are line assignments between points from two dynamical systems. Such line assignments are determined by a commuter, which is a coordinates transformation function that satisfies a commuting relationship but not necessarily a homeomorphism. The bundle structure is studied by understanding the implication of the system's qualitative changes. In addition, the case of the bundle plot with higher dimensional parameter variation is also considered. A main concern in the bundle plot is a special structure, called "joint", which determines a critical value of the parameter where the kneading sequence becomes periodic.
\end{abstract}

Keywords: Bundle plot; assignment plot; commuter; mostly conjugacy; kneading theory; unimodal map; symbolic dynamics; bifurcation theory.

\section{Introduction}

An interesting and fundamental question in science and dynamical systems is how do we describe evolution of systems due to parameter changes. Bifurcation theory characterizes qualitative changes in the way of tracking fixed points' creation, or destruction, or stability changes. A bifurcation occurs when a small change made to a system's parameter causes a topological difference in its behavior. So two similar systems with the same formation but slightly different parameter values may behave qualitatively different. Some "difference measurements" such as least square method fail to reveal the underlying topological distinction. Bollt and Skufca introduced a concept of a commuter to extend the equivalence relationship of conjugacy to measure the distance from being conjugate, which can also be thought of as the degree of "matching" between trajectories [Skufca \& Bollt, 2008; Zheng et al., 2011]. Later, they extended and interpreted the concepts of "commuter" and "defect measure" to symbol space, and introduced a new visualization technique, called assignment plot, to show matchings between symbol sequences of two topological spaces [Bollt \& Skufca, 2010].

Based on this perspective, we consider a "limit process" of assembling assignment plots from a set of different system parameter values. We extend here the idea of studying the qualitative difference between two systems to that within a family of 
systems, where we give the name "bundle plot" to the resulting structure. Compared to traditional bifurcation theory, instead of studying the qualitative changes of fixed points, we picture the symbolic space evolution due to continuous changes of system parameters. The bundle structure implies trajectories, or say symbolic sequences, creation or destruction, and the "speed" of such changes. In addition, a special structure "joint" implies that the kneading sequence is periodic, which happens shortly after the periodic window opening.

This paper is organized as follows: in Sec. 2, we review the concept of "mostly conjugacy" and "commuter"; in Sec. 3, we extend and interpret the concepts from a symbolic dynamics perspective; in Sec. 4, we extend the assignment plot to the bundle plot; we consider the bundle plot in higher dimensional parametric changes in Sec. 5; then we compare our result to that of bifurcation theory in Sec. 6; we complete our paper with discussion and future work in Sec. 7 .

\section{Comparing Dynamical Systems: Review of Nonhomeomorphic Commuter}

An essential question within the modeling context is "how close is the model to the true phenomena," where the natural system under consideration is dynamic, with possibly complex behavior. The field of dynamical systems seeks to provide an appropriate framework to study these systems. Since the inception of the field of dynamical systems by Henri Poincaré [Poincaré \& Magini, 1899], the fundamental approach has been to examine topological and geometric features of orbits, rather than focusing on numerical specifics of particular solutions of the dynamical system, as measured in some specific coordinate system. Characterization of the system relies on deciphering coordinate independent properties, such as the periodic orbit structure - the count and stability of periodic orbits.

In dynamical systems, the usual way to relate two dynamical systems is with the topological notion of conjugacy, related by the following commuting diagram,

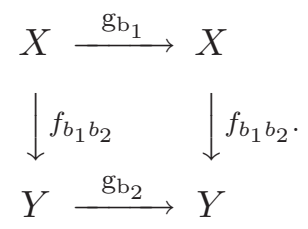

The conjugacy function $f_{b_{1} b_{2}}: X \rightarrow Y$ satisfies the commuting relationship

$$
f_{b_{1} b_{2}} \circ g_{b_{1}}=g_{b_{2}} \circ f_{b_{1} b_{2}},
$$

with $b_{i}$ the parameter of system $g_{b_{i}}$. The conjugacy $f_{b_{1} b_{2}}$ is a homeomorphism from system $g_{b_{1}}$ to $g_{b_{2}}$, i.e. $f_{b_{1} b_{2}}$ is 1-to- 1 , onto, continuous and has a continuous inverse function. It is the change of coordinates that the mappings behave exactly the same in either coordinate system. However, a commuter is an arbitrary function, not necessarily a homeomorphism, that satisfies the commuting relationship, in which case it is a nonhomeomorphic change of coordinates translating between dissimilar systems. Note the commuter provides a matching between trajectories for $g_{b_{1}}$ and $g_{b_{2}}$ over- and/or under-representations are reflected as 1-to-1 and onto problems in $f$, while trajectories that permit matching only for finite time are related to discontinuities in $f$. We therefore developed measures of commuters $f$ to quantify "how much" $f$ fail to be a "perfect match", i.e. homeomorphism [Skufca \& Bollt, 2008; Zheng et al., 2011]. For two topological conjugate systems which are related by a homeomorphism, the dynamics of one system completely describe the dynamics of the other. Thus, the notion of "distance to conjugacy" should provide a means of determining the extent to which the dynamics are similar. Bollt and Skufca [2008] defined and studied the measure of the deviation from homeomorphism, named homeomorphic defect, which provides a weighted average based on measurements of possible failure of $f$ being onto, 1-1, continuous and inverse continuous. This defect measure is proven to have certain regularity properties [Zheng et al., 2011], which supports our definition of "distance".

On construction of commuter $f_{b_{1} b_{2}}$, we define a commuter operator $f_{b_{1} b_{2}}^{n+1}(x)=\mathfrak{C}_{g_{b_{1}}}^{g_{b_{2}}} f_{b_{1} b_{2}}^{n}(x)=$ $g_{b_{2}}^{-1} \circ f_{b_{1} b_{2}}^{n} \circ g_{b_{1}}(x)$, with $f_{b_{1} b_{2}}^{1}=I$. The subscript of $f_{b_{1} b_{2}}^{n}$ means this is a $n$-iterate commuter from system $g_{b_{1}}$ to $g_{b_{2}}$. We require $g_{b_{2}}^{-1}$ to be a well defined inverse function and piecewise Lipshitz continuous with constant $L<1$, which guarantees there is a unique fixed point, say $f_{b_{1} b_{2}}$, of the commutation operator $\mathfrak{C}_{g_{b_{1}}}^{g_{b_{2}}}$. The left column of Fig. 1 shows an example of commuters between a short logistic map and skew full tent map, and between skew full tent map and short logistic map. Notice that the vertical gaps of the first commuter shows that, there are some intervals of the full tent that cannot be matched by the short logistic. 


\section{A Symbolic Dynamics Interpretation of Commuter Functions}

The commuter function in the previous section describes a pointwise matching from one coordinate system to the other. We discuss the idea of a commuter in a symbolic dynamics interpretation, which illustrates the nonhomeomorphism in a broader setting. In particular, each point $x$ in $g_{b_{1}}$ is represented in a different coordinate system $g_{b_{2}}$ by $y=f_{b_{1} b_{2}}(x)$. The degree to which the commuter fails to be a homeomorphism defines what we call a homeomorphic defect. However, there were limits in the mathematical technology requiring that the transformations be one-dimensional mappings for rigorous construction of the commuters by fixed point iteration. Further, there are difficulties in numerically computing defects in the more complicated one-dimensional cases, and further limits to higher dimensional problems. Interpretation of dynamical systems through a symbolic representation has become the standard tool for identifying key dynamical structures and behaviors, particularly when studying chaotic systems [Ott, 2002]. Therefore, Bollt and Skufca [2010] extended the "commuter" theory to a symbolic dynamics setting, which allows for multivariate transformations, with construction methods separate from the fixed point iteration, and new methods to compute defect. In addition, we introduce assignment mappings/plots, which is a new visualization technique of commuters, to understand and illustrate commuters from a broader perspective (see Fig. 1).

In fact, the symbolic commuter matches points in $X$ to points in $Y$ such that the respective symbolic sequences will match for as many symbols as possible, which is proven in paper [Bollt \& Skufca, 2010]. This also provides us an optimization criterion to construct commuters for higher dimensional dynamical systems. In principle, we assume that the dynamical systems under consideration are presented to us with a known symbolic dynamic partitioning. In practise, if necessary, we can use the uniform partitioning to approximate the "actual" partition that can fully describe the original system, which is the focus in a later work [Zheng et al., 2012].

To define symbol dynamics for systems, we assume a shift space (or say grammar) on $\Sigma_{n}$, with integer symbols $1, \ldots, n$. We associate symbol $i$ with interval $I_{X_{i}}$ for the dynamics on $X$ and with $\left[y_{i-1}, y_{i}\right]$ for the dynamics on $Y$. A trajectory of systems $g_{b_{1}}$, given by $\left\{x, g_{b_{1}}(x), g_{b_{1}}^{2}(x), \ldots\right\}$ has an associated symbolic trajectory $S(x)=s_{0} s_{1}, \ldots$, where $g_{b_{1}}^{j}(x) \in I_{X_{i}} \Rightarrow s_{j}=i$. Similarly, a trajectory of system $g_{b_{2}}$, given by $\left\{y, g_{b_{2}}(y), g_{b_{2}}^{2}(y), \ldots\right\}$ has an associated symbolic trajectory $S(y)=s_{0} s_{1}, \ldots$, where $g_{b_{2}}^{j}(y) \in\left[y_{i-1}, y_{i}\right] \Rightarrow s_{j}=i$. We remark that because the closed partition on $Y$ gives an overlap at endpoints, the symbolic trajectory is nonunique for any preimage of any element of $E_{y}$. We denote $\Sigma^{y}$ as the subshift of all possible symbolic sequences for $y$.

The algorithm to construct symbolic commuter, which is given in detail in paper [Bollt \& Skufca, 2010], yields a unique commuter after one has assigned an appropriate partitioning from the spaces $X$ and $Y$. An assignment plot shows line segments from representative points in $X$, under $g_{12}$ to their images in $Y$. Figure 1 illustrates the characterization of the commuter as providing a matching between points/symbolic sequences/trajectories via lines.

In Fig. 1, we show assignment plots (blue lines) taken from the commuters between the maps on the left-hand side of the diagram. The top assignment plot is taken from the commuter between a subshift logistic map $g_{b_{1}}$ and a full shift skew-tent map $g_{b_{2}}$. The vertical gap of the commuter implies that $g_{b_{2}}$ has some dynamics that $g_{b_{1}}$ does not. For example, $g_{b_{1}}$ admits neither $a b a a$ nor bbaa, which can be observed from the first assignment plot, associated to the largest vertical gap in the first commuter. Similarly for the second assignment plot, $g_{b_{2}}$ is a full shift of two symbols, the words abaa and bbaa exist in the dynamics of $g_{b_{2}}$, but cannot be "matched" to a depth of four symbols with any point of $g_{b_{1}}$. Those intervals are associated to the largest horizontal portion of the second commuter. The commuter maps those points to 0.5 , on the boundary between symbols $a$ and $b$ of the $g_{b_{1}}$ dynamics, yielding a match to a depth of three symbols, either $a b a$ or $b b a$ as appropriate.

In the next section, we generalize the idea of assignment plot for comparison among a family of systems, named a bundle plot. A bundle plot can be regarded as an evolution of the shift space of a particular system, under the variation of a parameter. It shows how the system gains or loses dynamics/grammar when we increase or decrease the parameter. 


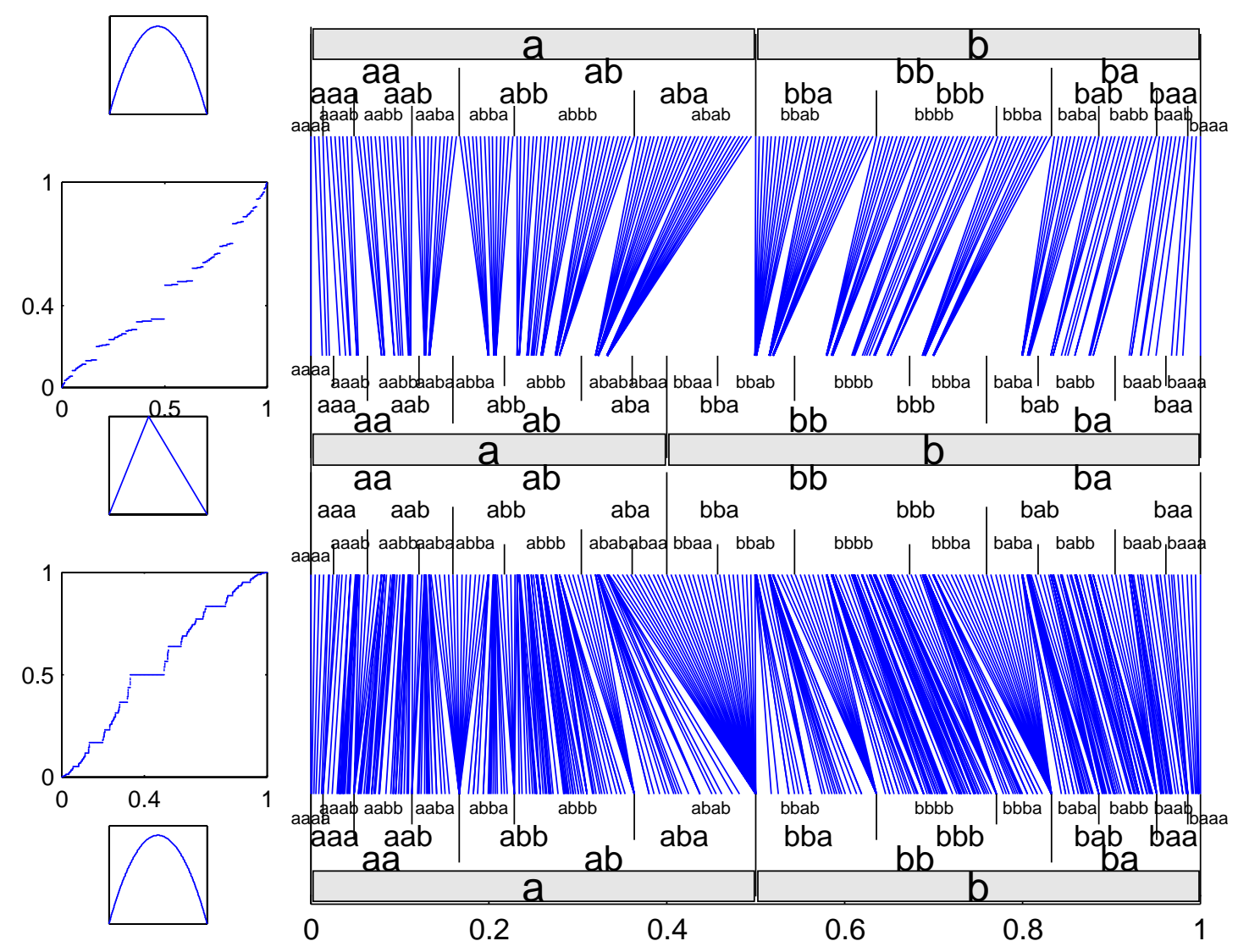

Fig. 1. Commuter as the matching of symbol dynamics. (Left column) A sequence of maps: $(1) g_{b_{1}}=3.6 x(1-x)$, a subshift logistic map; (2) the commuter between $g_{b_{1}}$ and (3) the full shift skew-tent map $g_{b_{2}} ;(4)$ the commuter from $g_{b_{2}}$ back (5) map $g_{b_{1}}$. (Right column) The symbol dynamic partition of the unit interval for maps $g_{b_{1}}$ (at top and bottom) and $g_{b_{2}}$ (in the middle), where we show all intervals for word length of four or fewer symbols. The assignment plot (blue lines) is taken from the commuters between the maps (as computed from the fixed point iteration), and shows that the commuter also gives a symbolic dynamic match between the two systems. Observe: (1) Map $g_{b_{1}}$ admits neither abaa nor bbaa, associated to the largest vertical gap in the first commuter. (2) Because $g_{b_{2}}$ is a full shift on two symbols, the words $a b a a$ and bbaa exist in the dynamics of $g_{b_{2}}$, but cannot be "matched" to a depth of four symbols with any point of $g_{b_{1}}$. Those intervals are associated to the largest horizontal portion of the second commuter. The commuter maps those points to 0.5, on the boundary between symbols $a$ and $b$ of the $g_{b_{1}}$ dynamics, yielding a match to a depth of three symbols, either $a b a$ or $b b a$ as appropriate.

\section{The Bundle: Assignment Plots of Commuters for a Family of Maps}

For a discrete dynamical system $x_{n+1}=g\left(x_{n}, b_{i}\right):=$ $g_{b_{i}}\left(x_{n}\right)$, where $b_{i}$ is the parameter of the system, we want to study the creation or destruction of allowable symbolic sequences/trajectories of the system as we vary $b_{i}$. We let $g_{b_{1}}$ be the base system with a particular fixed parameter $b_{1}$, and compare it to mappings $\left\{g_{b_{i}}\right\}$. Denote $f_{b_{1} b_{i}}$ to be the commuter from $g_{b_{1}}$ to $g_{b_{i}}$. Denote $f_{b_{1} b_{i}}^{n}$ to be the $n$-step commuter between $g_{b_{1}}$ and $g_{b_{i}}$ in the iteration scheme that generates the commuter. We let $f_{b_{1} b_{i}}^{1}$, i.e. the initial guess, to be $I$.

For each $x$ in the base space $X$, we line up $x$ to all the points of $\left\{f_{b_{1} b_{i}}(x)\right\}$, with $\left\{b_{i}\right\}$ values in an interval. Figure 2 illustrates such association from the family of tent maps across $0.5 \leq b_{i} \leq 1$, with $b_{1}=1$.

$x_{n+1}=g_{b_{i}}\left(x_{n}\right)= \begin{cases}2 b_{i} x_{n}, & 0 \leq x_{n} \leq \frac{1}{2}, \\ 2 b_{i}\left(1-x_{n}\right), & \frac{1}{2}<x_{n} \leq 1 .\end{cases}$

We call $g_{1}$, the full $\left(b_{1}=1\right)$ symmetric tent map, the base system. Each curve describes the matching between each $x$ and $\left\{f_{1 b_{i}}(x)\right\}$. We name the entire structure as "bundle", and each curve as a "fiber". The names of "bundle" and "fiber" here are consistent with the concept of bundles in Topology, 


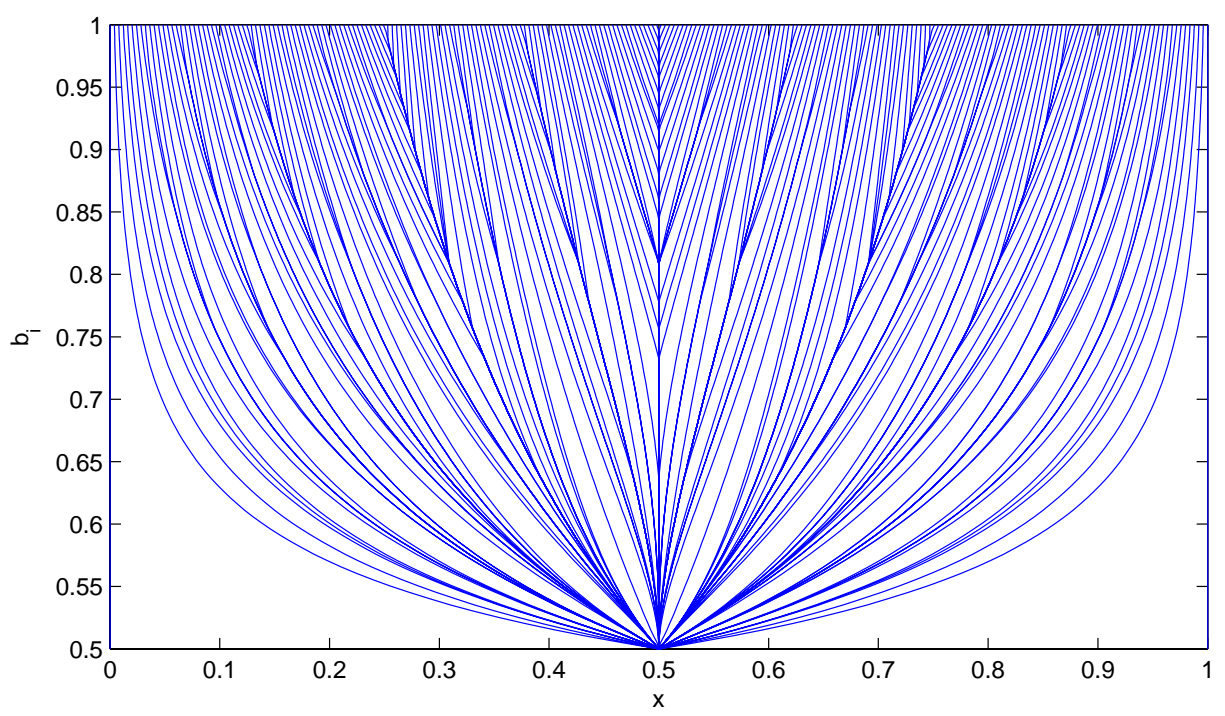

Fig. 2. A bundle plot of one-dimensional mappings $f_{1 b_{i}}: X \rightarrow Y$, with the domain $X=[0,1]$ shown at the top, and the range $Y \subset[0,1]$. Line segments show assignments from $X$ to each $Y$ of a uniform grid of sample points representing the commuters $f_{1 b_{i}}$ from $g_{b_{1}}$ and $g_{b_{i}}$, where $g_{b_{1}}$ is the full symmetric tent map and $g_{b_{i}}$ is short symmetric tent map with height $b_{i} \in[1 / 2,1]$.

as we cite the definition below:

Definition 4.1 (Fiber bundle [Rowland]). A fiber bundle (also called simply a bundle) with fiber $F$ is a map $f: E \rightarrow B$ where $E$ is called the total space of the fiber bundle and $B$ the base space of the fiber bundle. The main condition for the map to be a fiber bundle is that every point in the base space $b \in B$ has a neighborhood $U$ such that $f^{-1}(U)$ is homeomorphic to $U \times F$ in a special way. Namely, if

$$
h: f^{-1}(U) \rightarrow U \times F
$$

is the homeomorphism, then

$$
\operatorname{proj}_{U} \circ h=f_{\left|f^{-1}(U)\right|},
$$

where the map $\operatorname{proj}_{U}$ means projection onto the $U$ component. The homeomorphisms $h$ which "commute with projection" are called local trivializations for the fiber bundle $f$. In other words, $E$ looks like the product $B \times F$ (at least locally), except that the fibers $f^{-1}(x)$ for $x \in B$ may be a bit "twisted".

So following this definition, if we let $B=$ $\left\{b_{i}\right\}, F=\left\{f_{1 b_{i}}(x)\right\}$, then $E=B \times F$ is a trivial fiber bundle.

In Fig. 2, we can see that some of the fibers merge to singletons as we decrease the height of the tent map. A similar story happens for a family of symmetric logistic maps (Fig. 3), i.e. $\left\{g_{b_{i}}(x)\right.$ : $\left.x_{n+1}=g_{b_{i}}\left(x_{n}\right)=4 b_{i} x_{n}\left(1-x_{n}\right)\right\}$ with the height $0.89<b_{i}<1$ and $b_{1}=1$. Note $b_{i}>0.89$ guarantees the existence of the commuter between $g_{b_{1}}$ and $g_{b_{i}}$.

Here we are only concerned about "symbolic fibers" $\left\{f_{1 b_{i}}(x)\right\}$ where $x$ are the generating partition boundary points. In particular,

Definition 4.2. Let $\mathscr{P}=\left\{x \mid g_{b_{1}}^{n}(x)=1 / 2\right\}$, the set of all preimages of $1 / 2$ of $g_{b_{1}}(x)$. The symbolic fibers form the set $F=\left\{\left\{f_{1 b_{i}}(x)\right\} \mid x \in \mathscr{P}\right\}$.

A singleton from mergence of symbolic fibers at $b$ simply implies $\exists x_{1}, x_{2} \in \mathscr{P}, x_{1} \neq x_{2}$ with $\left\{f_{1 b_{i}}\left(x_{1}\right)\right\}=\left\{f_{1 b_{i}}\left(x_{2}\right)\right\}, \forall b_{i} \geq b$. The fibers from nongenerating partitioning end points are "bounded" by two symbolic fibers and do not cross each other.

In the following, we study the bundle and those symbolic fibers in terms of symbolic dynamics. We shall start our discussion of bundles in terms of the tent map and hopefully it is apparent that other maps can be handled similarly. As can be observed from Fig. 2, we identify some basic "branch" structures which assemble the entire bundle plot. As roughly sketched in Fig. 4, we investigate the "branch" structure as three elemental structures, which are demonstrated in Fig. 4(top). Top left shows that one symbolic fiber on each side of the mid-vertical fiber join together; Top middle shows only one symbolic fiber that from one side joins the mid fiber; while the top right has two symbolic fibers on each side of mid fiber joining together. 


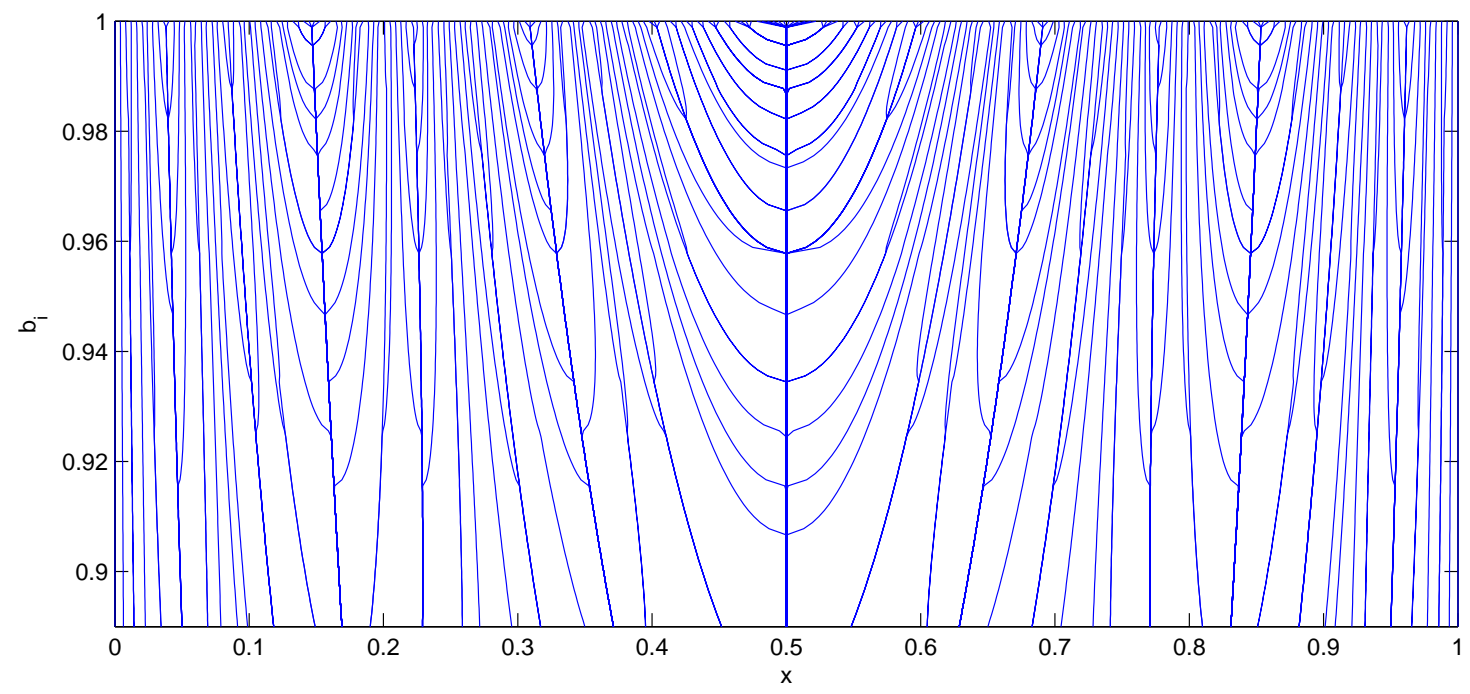

Fig. 3. A bundle plot of one-dimensional mappings $f_{1 b_{i}}: X \rightarrow Y$, with the domain $X=[0,1]$ shown at the top, and the range $Y \subset[0,1]$. Line segments show assignments from $X$ to each $Y$ of a uniform grid of sample points representing the commuters $f_{1 b_{i}}$ from $g_{b_{1}}$ and $g_{b_{i}}$, where $g_{b_{1}}$ is the full logistic map and $g_{b_{i}}$ is the short logistic map with height $b_{i} \in[0.89,1]$.

The whole bundle structure is a fractal structure such that any elemental structure repeats others including itself. In the following, we will theoretically interpret the elemental structures in terms of symbolic dynamics.
We first focus on the top left elemental structure in Fig. 4. As we decrease the height of the tent map, the system loses dynamics by losing allowable symbolic sequences. Suppose we have a generating partition, which gives four intervals

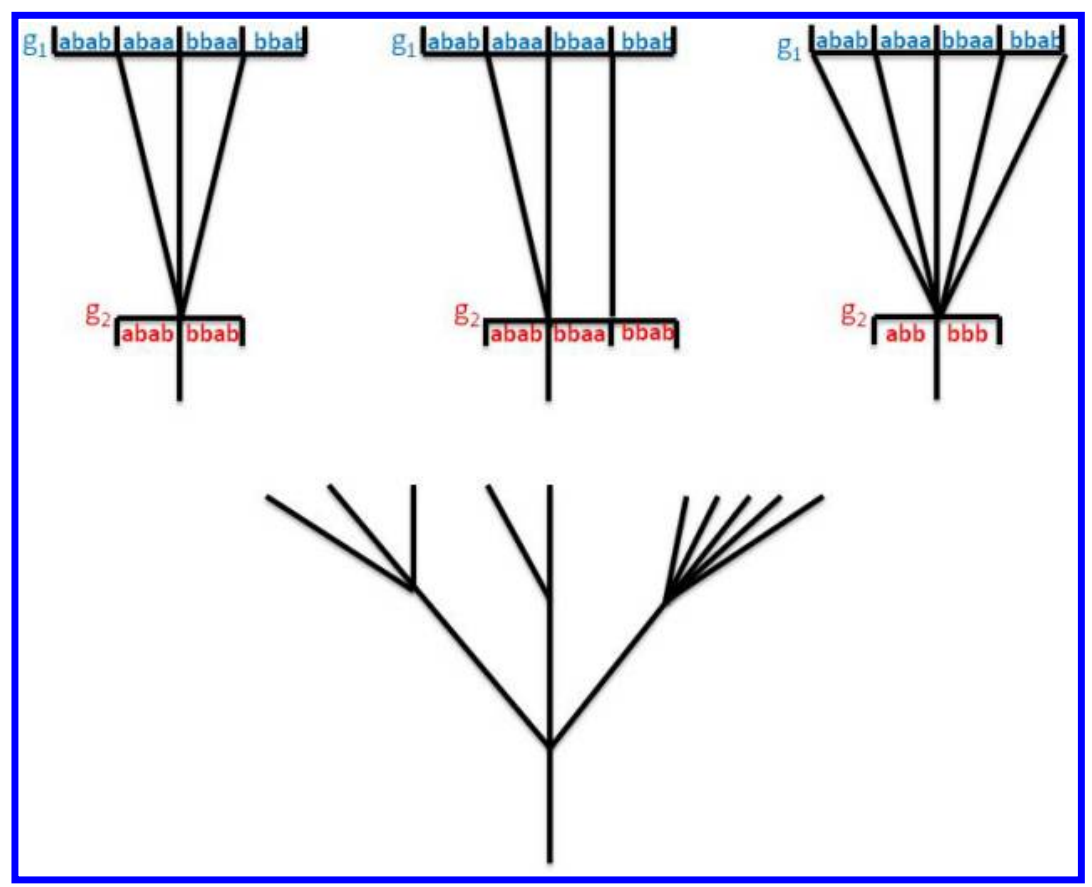

Fig. 4. Elemental structure of a bundle: Suppose we have a generating partition, which consists of these four intervals $a b a b, a b a a, b b a a, b b a b$ in $g_{b_{1}}$ system. (Top left) For the system $g_{b_{2}}$, suppose the sequences $a b a a$ and $b b a a$ are not allowable, the intervals $a b a a$ and $b b a a$ in $g_{b_{1}}$ map to a singleton of $g_{b_{2}}$. We called this case to be "losing 2 words of length 4 "; (Top middle) Only an interval on the left-hand side of the mid fiber loses 1 word, while the right-hand side still has a perfect matching; (Top right) $b b a a, b b a b, a b a a$ and $a b a b$ (or say the entire $a b a$ and $b b a$ ) are lost at the same time, which describes a "loss of 4 words of length 4, or loss of 2 words of length 3". (Bottom) The entire bundle plot is assembled by the top three elemental structures. 
$a b a b, a b a a, b b a a, b b a b$ in $g_{b_{1}}$ system. Then, for instance, the points in the interval $a b a b$ has an infinitely long sequence and starting with the word $a b a b$. Now suppose we compare $g_{b_{1}}$ to itself (Fig. 6, left column). The commuter is trivially the identity maps, meaning every symbolic sequence matches itself perfectly ("perfect" means the commuter is a homeomorphism); But as we lower the height of $g_{b_{2}}$ below 1 (Fig. 6 , mid column, the red tent map is lower), the $a b a a$ and bbaa intervals are compressed, since they lose allowable symbolic sequences starting with $a b a a$ or bbaa due to loss of dynamics of the $g_{b_{2}}$ system; Until a critical value of $b$, the $a b a a$ and bbaa intervals completely disappear, and the $a b a a$ and bbaa of $g_{b_{1}}$ map to a singleton of $g_{b_{2}}$. We simply call this case to be "losing 2 words of length 4", in the sense that we lose the word $a b a a$ and bbaa at the same time; In fact, in this case, we lose the word baa. For this, we notice when we lower the height of tent map $g_{b_{2}}$, the invariant set becomes smaller and excludes the interval baa, which appears as the right most interval under the generating partition [see Fig. 5(a)]. Thus the system loses the ability to generate any word with baa included, like $a b a a$ and $b b a a$. Therefore the words abaa and bbaa are not allowable in the grammar. What is more important is, we lose both words simultaneously. A step further, the loss of baa implies the associated kneading sequence is periodic (which will be proven in Sec. 6). For example, Fig. 5(a) shows the tent map bundle plot on the generating partition up to four symbols, with the red line at around 0.809 crossing a singleton, which is from the fibers of the left end point of $a b a a$ and right end point of bbaa. Consider the tent map with that associate height (around 0.809), whose kneading sequence happens to be period-3 with $\overline{C b a}$ (or say $g_{0.809}\left(g_{0.809}\left(g_{0.809}(0.5)\right)\right)=0.5$ ). If we start with an initial point slightly greater than 0.5 [see Fig. 5(b)], we can only have the symbolic sequence $b b a b \ldots .$. In other words, bbaa... is impossible. Similarly if we start with an initial point slightly less than 0.5 , we only have $a b a b$. But if we have the tent map higher than 0.809 , both $a b a a$ and $b b a a$ are allowable. For the family of symmetric tent map, the loss of words with certain length can be in pairs.

The top right structure in Fig. 4, however, describes a "loss of 4 words of length 4", which is due to the fact that $a b a b, a b a a, b b a a$ and $b b a b$ are lost at the same time. This correspond to a "faster" loss of words comparing to the case of "losing 2 words of length 4".

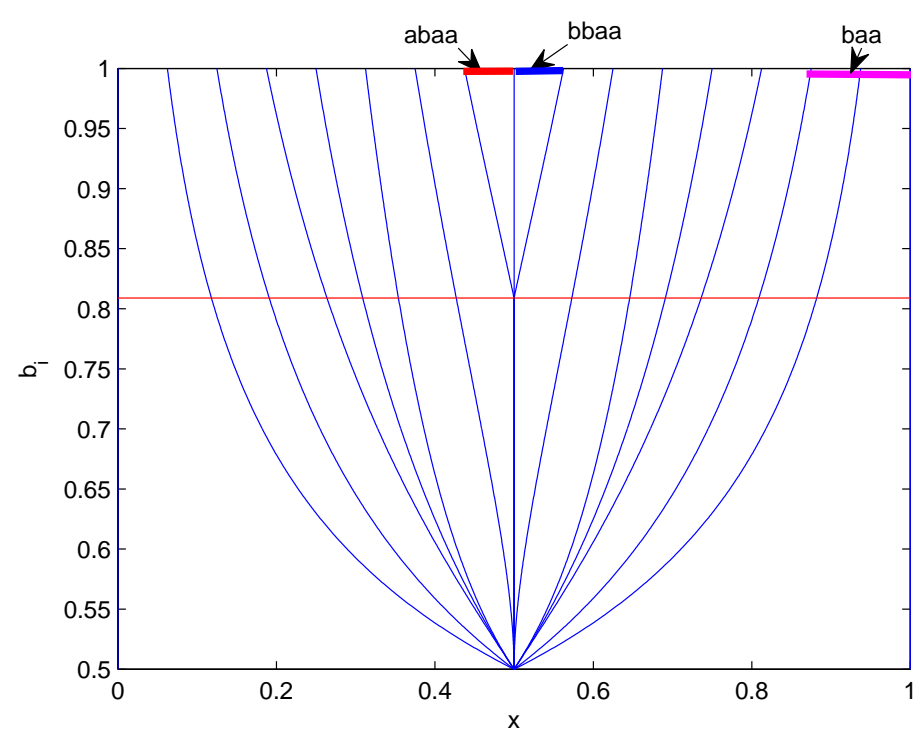

(a)

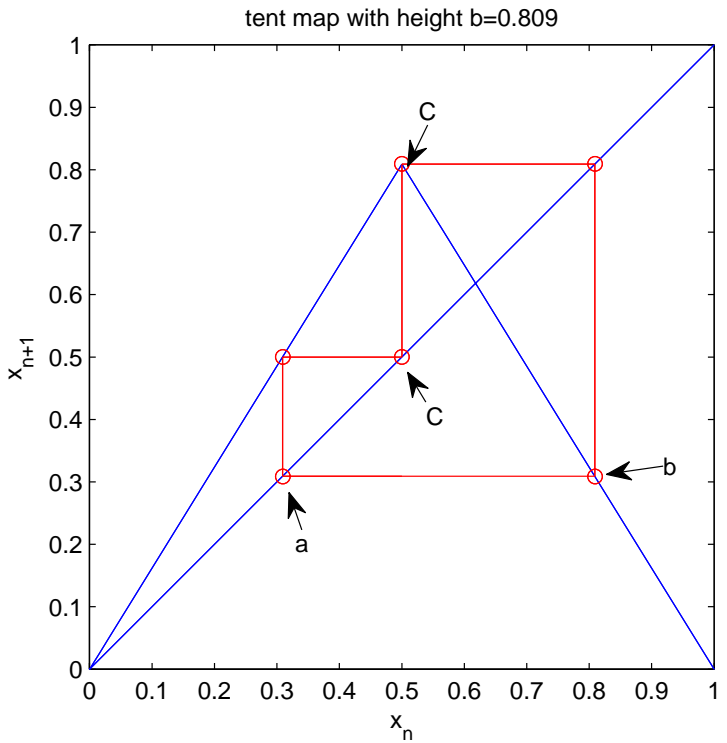

(b)

Fig. 5. Illustration of loss of 2 words of length 4: (a) The tent map bundle plot on the generating partition up to four symbols, with the red line at around 0.809 crossing a singleton, which is from the fibers of the left end point of $a b a a$ and right end point of bbaa. The singleton across the red line implies the loss of words abaa and bbaa, which further implies the loss of the word $b a a$. This is due to the fact that the invariant set of tent map at height 0.809 excludes the interval baa, and thus $a b a a$ and bbaa are not allowable in the grammar; (b) The tent map with associate height (around 0.809), whose kneading sequence happens to period-3 with $\overline{C b a}$ (or say $\left.g_{0.809}\left(g_{0.809}\left(g_{0.809}(0.5)\right)\right)=0.5\right)$. Note both $a b a a$ and bbaa ... are not allowable under the system. 


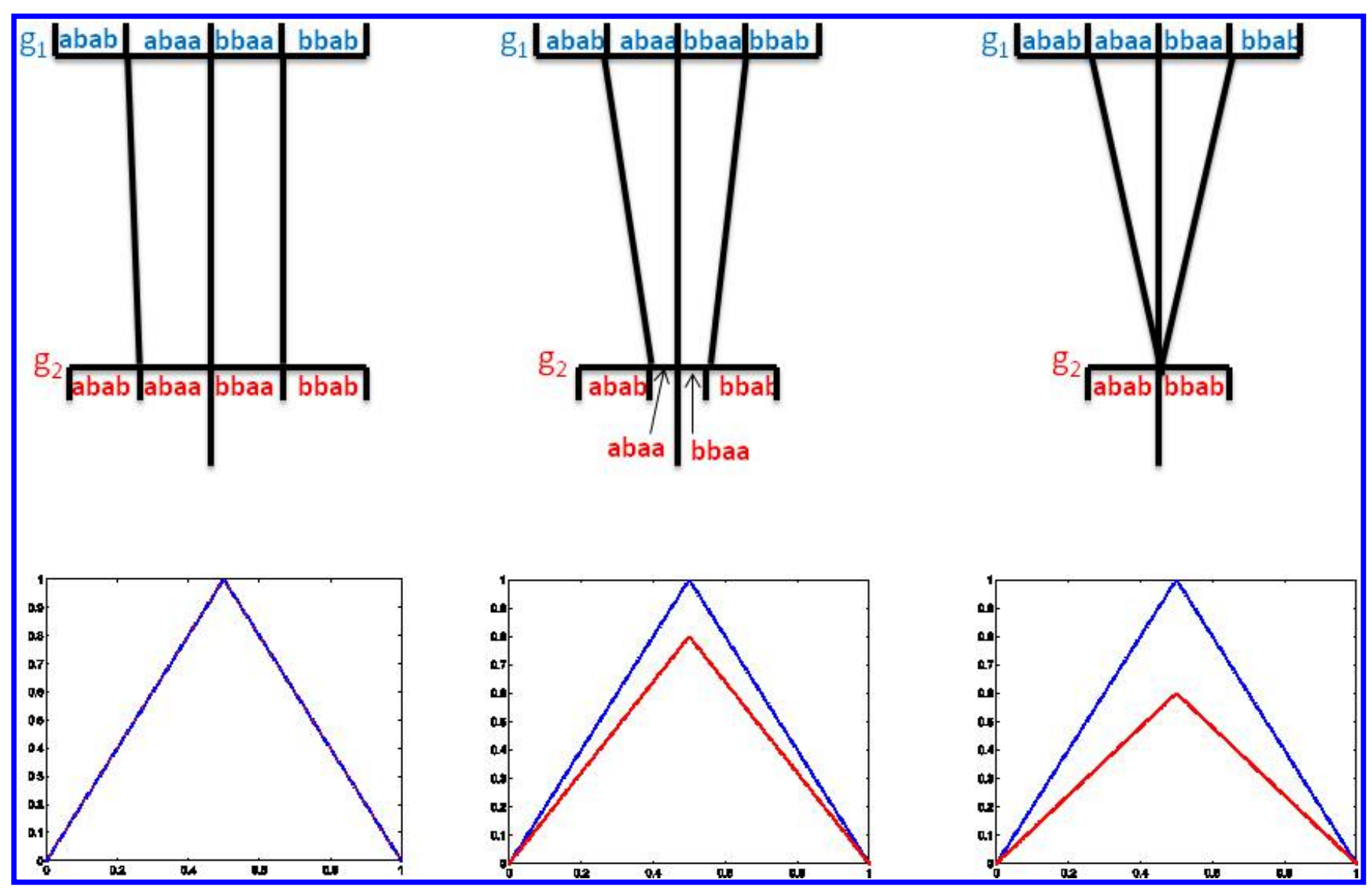

Fig. 6. Degeneration of intervals: (Left column) Suppose we compare $g_{b_{1}}$ to itself, the commuter is trivially the identity maps, meaning every symbolic sequence matches itself perfectly; (Middle column) As we lower the height of $g_{b_{2}}$ below 1 , the $a b a a$ and bbaa intervals are compressed; (Right column) At a critical value of $b$, the $a b a a$ and bbaa intervals completely disappear, the $a b a a$ and bbaa in $g_{b_{1}}$ map to a singleton of $g_{b_{2}}$.

Based on these observations and analysis, we call the singleton a "joint", and define its associate parameter $b$ as follows:

Definition 4.3 [Joint]. A joint parameter is denoted as

$$
\begin{aligned}
b_{\text {joint }}(\omega)= & \sup \{b \mid \text { the grammar } \Sigma \text { loses a word } \\
& \omega \text { of length } N\} .
\end{aligned}
$$

As long as there is no confusion, we label joint as the topological behavior and the associated parameter values. Whenever there is no need to distinguish $b_{\text {joint }}\left(\omega_{1}\right)$ from $b_{\text {joint }}\left(\omega_{2}\right)$, we simplify the notation $b_{\text {joint }}(\omega)$ as $b_{\text {joint }}$. In this paper, we are concerned about problems on the joint: When does joint happen? Is there any relationship between the joints and the bifurcation? Generally speaking, when we decrease the height of the tent maps or logistic maps, the complexity and entropy of the systems would decrease, due to the loss of periodic orbits. For instance, in Fig. 8, we calculate the total number of fibers for each $b_{i}$, which, as expected, gives a monotone increasing function, as we increase $b_{i}$ towards 1 . As we increase the size of the grid points in $X$ space and $b$ space, the "flat spot" and

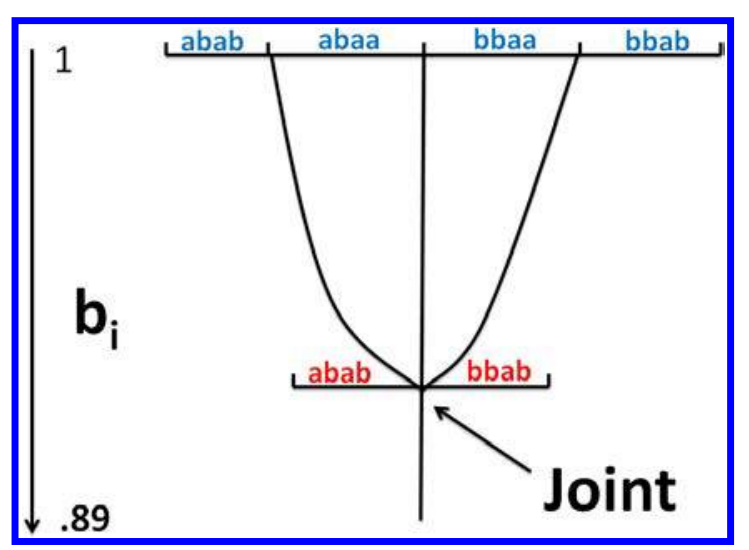

Fig. 7. Joint: the existence of a joint implies loss of a word/words. 


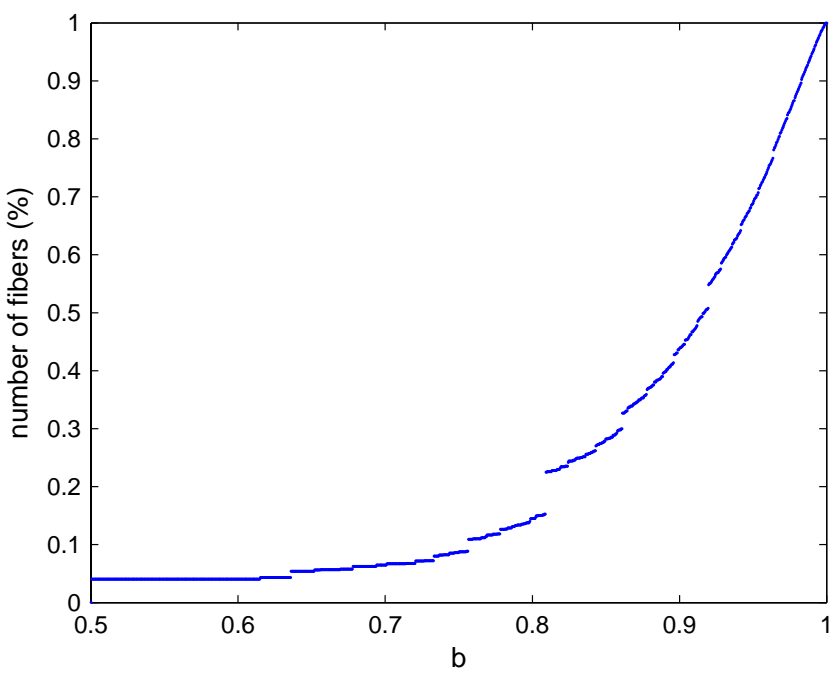

Fig. 8. Fibers' number: As we decrease the height of the tent map, the system loses dynamics in the way of losing allowable symbolic sequences. We calculate the total number of fibers for each $b_{i}$.

"jump" in Fig. 8 still exist, because once a particular symbolic sequence is lost, all the symbolic sequences of its preimage disappear simultaneously. Thus, as we decrease the value of $b_{i}$, we may lose symbolic sequences that are already lost.

In particular, the following theorem proves that the loss of symbolic sequences are actually nonsmooth. More precisely, it can be represented as a Lebesgue singular function. In other words, the derivative of commuters with respect to parameter $b$ is not a smooth function, but 0 almost everywhere (though it is monotone nondecreasing from 0 to 1 ). We introduce one theorem and two lemmas to prove this claim.

Theorem 1. The derivative of commuter with respect to the model parameter $b$ exists almost everywhere.

Proof. Since the commuter function is a piecewise monotone function with respect to parameter $b$ [Skufca \& Bollt, 2008; Zheng et al., 2012], and hence of bounded variation. Thus its derivative exists a.e. [Wheeden \& Zygumund, 1977].

Lemma 1. $f_{1 b}^{n}\left(x_{0}\right) \rightarrow f_{b}\left(x_{0}\right)$ for all $x_{0} \in[0,1]$ as $n \rightarrow \infty$.

For the proof of this lemma, refer to our former work [Skufca \& Bollt, 2008]. It shows the existence and uniqueness of the commuter function by contraction mapping theorem.
Lemma 2. $f_{1 b_{i}}^{n}\left(x_{0}\right) \rightarrow f_{1 b}^{n}\left(x_{0}\right)$ uniformly for $x_{0} \in$ $[0,1]$ as $b_{i} \rightarrow b$.

Proof. For $x_{0} \in[0,1 / 2]$, we want to show, for $\forall \epsilon, \exists \delta$, for all $\left|b_{i}-b\right|<\delta$, we have $\mid f_{1 b_{i}}^{n}\left(x_{0}\right)-$ $f_{1 b}^{n}\left(x_{0}\right) \mid<\epsilon$.

We show by induction:

For $n=1$, since $f_{1 b_{i}}^{1}=f_{1 b}^{1}=I$, the uniform convergence holds.

Now assume the case $n$ is true, that is $\forall \epsilon, \exists \delta$, for all $\left|b_{i}-b\right|<\delta$, we have $\left|f_{1 b_{i}}^{n}\left(x_{0}\right)-f_{1 b}^{n}\left(x_{0}\right)\right|<\epsilon$.

For the case $n+1$, since $f_{1 b_{i}}^{n+1}\left(x_{0}\right)=g_{b_{i}}^{-1} \circ f_{1 b_{i}}^{n} \circ$ $g_{1}\left(x_{0}\right)=\frac{1}{2 b_{i}} f_{1 b_{i}}^{n}\left(2 x_{0}\right)$, for the above $\epsilon$ in case $n$, $\exists \delta=\epsilon$, so that:

$$
\begin{aligned}
\left|f_{1 b}^{n+1}\left(x_{0}\right)-f_{1 b_{i}}^{n+1}\left(x_{0}\right)\right| \\
=\left|\frac{1}{2 b} f_{1 b}^{n}\left(2 x_{0}\right)-\frac{1}{2 b_{i+1}} f_{1 b_{i}}^{n}\left(2 x_{0}\right)\right| \\
\leq \frac{1}{2 b}\left|f_{1 b}^{n}\left(2 x_{0}\right)-f_{1 b_{i}}^{n}\left(2 x_{0}\right)\right| \\
\quad+\left|\frac{1}{2 b}-\frac{1}{2 b_{i}}\right| f_{1 b_{i}}^{n}\left(2 x_{0}\right) \\
<\frac{\epsilon}{2 b}+\frac{\epsilon}{2 b b_{i}}=\left(\frac{1}{2 b}+\frac{1}{2 b b_{i}}\right) \epsilon<3 \epsilon .
\end{aligned}
$$

For $x_{0} \in[1 / 2,1]$, the proof is similar.

Note that we are considering $b_{i}, b \in[1 / 2,1]$.

Theorem 2. The derivative of the commuter with respect to the parameter $b$ is 0 whenever it exists.

Proof. For $x_{0} \in\left(0, \frac{1}{2}\right)$, we let $H^{\prime}=\lim _{b_{2} \rightarrow b_{3}} \times$ $\frac{\left|f_{1 b_{2}}\left(x_{0}\right)-f_{1 b_{3}}\left(x_{0}\right)\right|}{\left|b_{2}-b_{3}\right|}$. We want to show $H^{\prime}=0$ whenever it exists.

Denote $\left(H^{n}\right)^{\prime}=\lim _{b_{2} \rightarrow b_{3}} \frac{\left|f_{1 b_{2}}^{n}\left(x_{0}\right)-f_{1 b_{3}}^{n}\left(x_{0}\right)\right|}{\left|b_{2}-b_{3}\right|}$, then by Lemmas 1 and 2, we can interchange the limit and have

$$
\begin{aligned}
H^{\prime} & =\lim _{b_{2} \rightarrow b_{3}} \lim _{n \rightarrow \infty} \frac{\left|f_{1 b_{2}}^{n}\left(x_{0}\right)-f_{1 b_{3}}^{n}\left(x_{0}\right)\right|}{\left|b_{2}-b_{3}\right|} \\
& =\lim _{n \rightarrow \infty} \lim _{b_{2} \rightarrow b_{3}} \frac{\left|f_{1 b_{2}}^{n}\left(x_{0}\right)-f_{1 b_{3}}^{n}\left(x_{0}\right)\right|}{\left|b_{2}-b_{3}\right|} \\
& =\lim _{n \rightarrow \infty}\left(H^{n}\right)^{\prime} .
\end{aligned}
$$

We show $\left(H^{n}\right)^{\prime}=0$ by induction. 
For that case when $n=1$, since $f_{1 b_{2}}^{1}=f_{1 b_{3}}^{1}=I$, the conclusion holds.

Assume the case $n$ is true, that is $\left(H^{n}\right)^{\prime}=0$ whenever it exists.

For the case $n+1,\left(H^{n+1}\right)^{\prime}=\lim _{b_{2} \rightarrow b_{3}} \times$ $\frac{\left|f_{1 b_{2}}^{n}\left(x_{0}\right)-f_{1 b_{3}}^{n}\left(x_{0}\right)\right|}{\left|b_{2}-b_{3}\right|}, x_{0} \in[0,1]$. Since $f_{1 i}^{n+1}=g_{b_{i}}^{-1} \circ$ $f_{1 b_{i}}^{n} \circ g_{b_{1}}$, we have

$$
\begin{array}{r}
\left(H^{n+1}\right)^{\prime}=\lim _{b_{2} \rightarrow b_{3}} \frac{\left|\frac{1}{2 b_{2}} f_{1 b_{2}}^{n}\left(x_{0}\right)-\frac{1}{2 b_{3}} f_{1 b_{3}}^{n}\left(x_{0}\right)\right|}{\left|b_{2}-b_{3}\right|}, \\
x_{0} \in\left[0, \frac{1}{2}\right]
\end{array}
$$

Note the proof for $x_{0} \in[1 / 2,1]$ is similar.

Without loss of generality, we assume $\frac{1}{2 b_{2}} f_{b_{1} b_{2}}^{n}\left(x_{0}\right)>\frac{1}{2 b_{3}} f_{b_{1} b_{3}}^{n}\left(x_{0}\right)$ and $b_{2}>b_{3}$, then we have

$$
\begin{aligned}
& \frac{\frac{1}{2 b_{2}}\left(f_{1 b_{2}}^{n}\left(2 x_{0}\right)-f_{1 b_{3}}^{n}\left(2 x_{0}\right)\right)}{b_{2}-b_{3}} \\
& \quad \leq \frac{\frac{1}{2 b_{2}} f_{1 b_{2}}^{n}\left(2 x_{0}\right)-\frac{1}{2 b_{3}} f_{1 b_{3}}^{n}\left(2 x_{0}\right)}{b_{2}-b_{3}} \\
& \quad \leq \frac{\frac{1}{2 b_{3}}\left(f_{1 b_{2}}^{n}\left(2 x_{0}\right)-f_{1 b_{3}}^{n}\left(2 x_{0}\right)\right)}{b_{2}-b_{3}} .
\end{aligned}
$$

We take the limit of $b_{2} \rightarrow b_{3}$ on the above inequality, and by assumption of case $n$, we have $\left(H^{n+1}\right)^{\prime}=0$. We note that for the assumption in case $n$, the conclusion is true for $x_{0} \in[0,1]$. While in case $n+1$, we are actually considering $\left(H^{n+1}\right)^{\prime}\left(2 x_{0}\right), x_{0} \in[0,1 / 2]$. But the measure of $x_{0}$ for $\left(H^{n}\right)^{\prime} \neq 0$ is 0 by Theorem 1 . So in other words, $\left(H^{n}\right)^{\prime}=0$ a.e. for $x_{0} \in[0,1]$, which is what we want to show.

Now we have proven that the variation of system parameter leads to a nonsmooth reduction of the system's dynamics. A typical symptom is the joint of fibers, which indicates the "loss of words" of some length. This gives rise to homeomorphic defect since some symbolic sequences of the base system cannot be matched. In the next section, we give another type of bundle, which we call "conjugate/skew bundle". Still, we vary the parameter of the system, but it only exhibits a homeomorphic change of the system. In other words, the bundle plot draws associations within the conjugate classes of the system, with no joint and no symbolic sequences lost.

\subsection{Skew bundle}

In the previous section, we consider the loss of symbolic sequences as we vary one parameter of the system. In this section, we are considering the bundle plot for a family of equivalent systems where all the systems are conjugate. We use the tent map as an example. It is known [Skufca \& Bollt, 2008], that skew tent maps with height 1 are conjugate systems. We fixed the heights of the tent maps $b_{i} \equiv 1$, while change the peak point's $x$-axis $a_{i}$ from 0 to 1 . In particular, the family of skew tent maps is given by the following:

$$
\begin{aligned}
x_{n+1} & =g_{a_{i}}\left(x_{n}\right) \\
& = \begin{cases}\frac{1}{a_{i}} x_{n}, & 0 \leq x_{n} \leq \frac{1}{2}, \\
\frac{1}{1-a_{i}}\left(1-x_{n}\right), & \frac{1}{2}<x_{n} \leq 1 .\end{cases}
\end{aligned}
$$

As shown in Fig. 9, the skew bundle exhibits a discontinuity that said colloquially, reminds us of a "side-parted hairstyle", with a single hair separating two sides. The fibers do not intersect each other, which indicate there is no loss of symbolic sequences of the system. Notice that the bundle plot in Fig. 9 is not symmetric and, unlike the case in the previous section, the vertical gaps of commuters [see Figs. 9(a) and 9(b)] do not mean there are homeomorphic defects. In fact, in this case the commuters are singular functions [Skufca \& Bollt, 2008].

There are no joints except at $a_{i}=0$ and $a_{i}=1$, whose tent maps have only one leg. For $0<a_{i}<1$, the tent map changes under conjugacies, as Figs. 9(a) and 9(b) show two particular conjugacies, which are between the symmetric tent map and skew tent maps.

The nonsymmetric structure of the bundle plot in Fig. 9 is due to the stable fixed point's location. We solve the nontrivial fixed point from $x^{*}=$ $\frac{1}{1-a}\left(1-x^{*}\right)$, and have

$$
a=2-\frac{1}{x^{*}}, \quad x^{*}>\frac{1}{2} .
$$




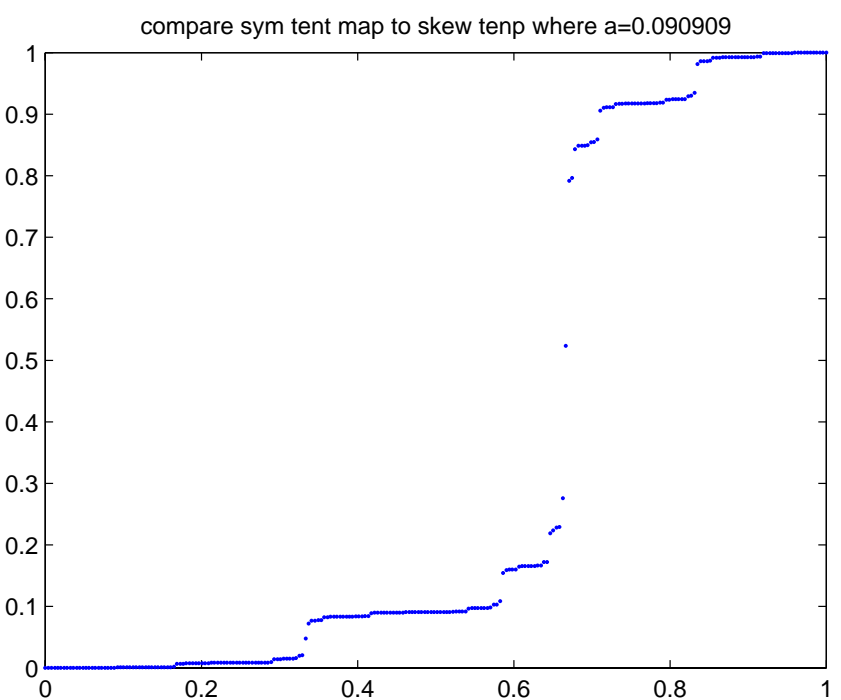

(a)

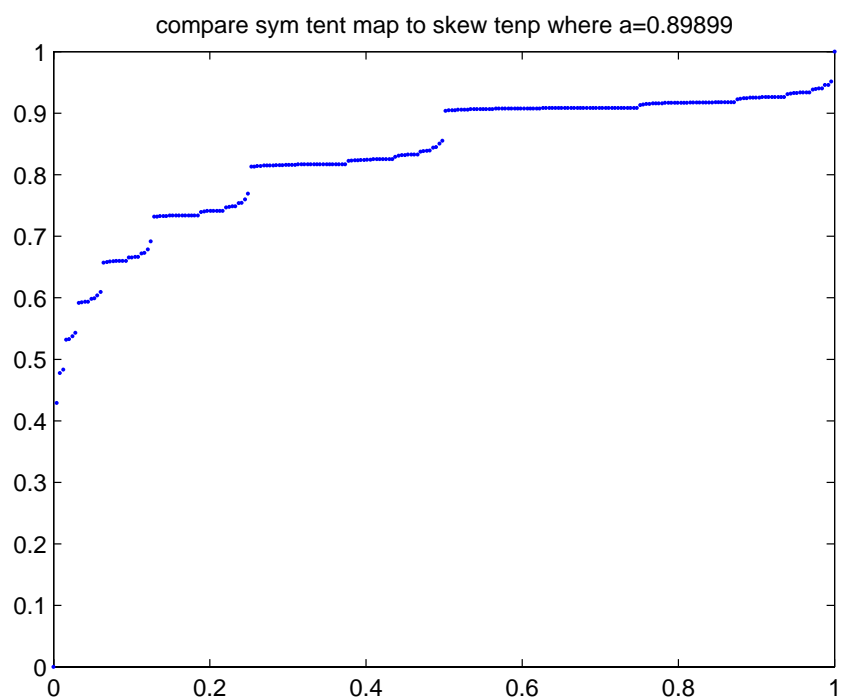

(b)

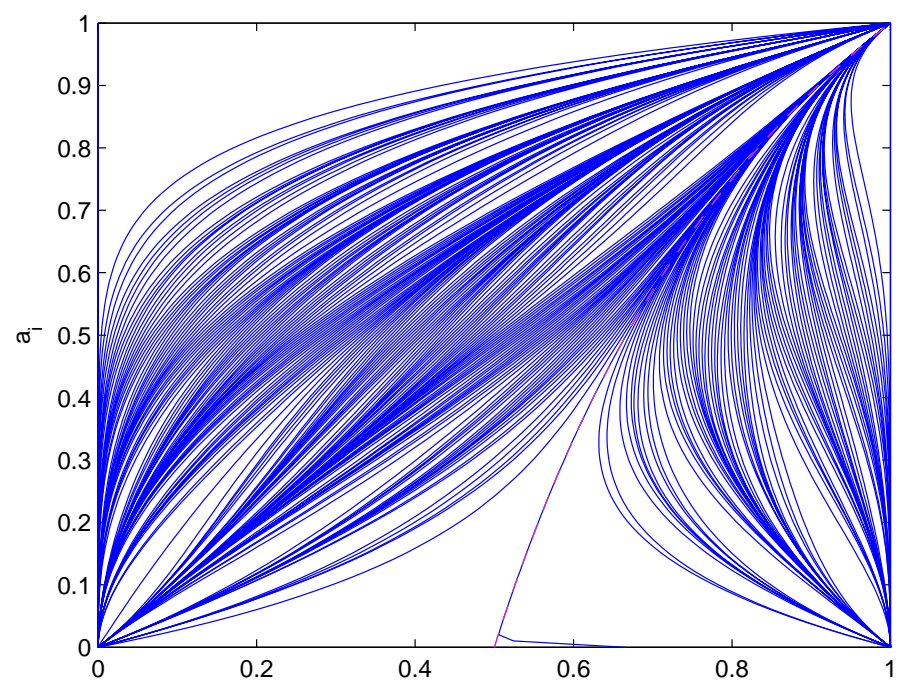

(c)

Fig. 9. A bundle plot for skew tent maps: Line segments show assignments from $X$ to each $Y$ of a uniform grid of sample points representing the commuters $f_{1 a_{i}}$ from $g_{a_{1}}$ to $g_{a_{i}}$, where $g_{a_{1}}$ is the full symmetric $\left(a_{1}=1 / 2\right)$ tent map and $g_{a_{i}}$ is skew tent maps with peak points at $\left(a_{i}, 1\right)$. (a) The commuter between the full symmetric tent map and a skew tent map with peak at $(0.090909,1)$; (b) The commuter between the full symmetric tent map and a skew tent map with peak at $(0.890909,1)$; (c) The skew bundle, which exhibits a "side-parted hairstyle", with a single hair, which is the stable fixed points' location for different $g_{a_{i}}$.

We plot it with red dash line in Fig. 9(c), which is exactly the "single hair". Points $x<1 / 2$ of $g_{a_{1}}$ match points $x<a_{i}$ of $\left\{g_{a_{i}}\right\} ; 1 / 2<x<x^{*}$ of $g_{a_{1}}$ match points $a_{i}<x<x^{*}$ of $\left\{g_{a_{i}}\right\} ; x>x^{*}$ of $g_{a_{1}}$ match those of $\left\{g_{a_{i}}\right\}$; While the fixed point $x^{*}$ of $g_{a_{1}}$ matches to those of $\left\{g_{a_{i}}\right\}$. As $a_{i}$ moves from 0 to 1 , the stable fixed point moves from $1 / 2$ to 1 , which skews the bundle to the right.

\section{Bundle for Higher Dimensional Parameter Space}

Here we consider the bundle plot where we change two parameters of the system simultaneously, which gives a three-dimensional bundle. For instance, Figs. 10 and 11 show bundle plots for comparing full symmetric tent map and tent maps with different 

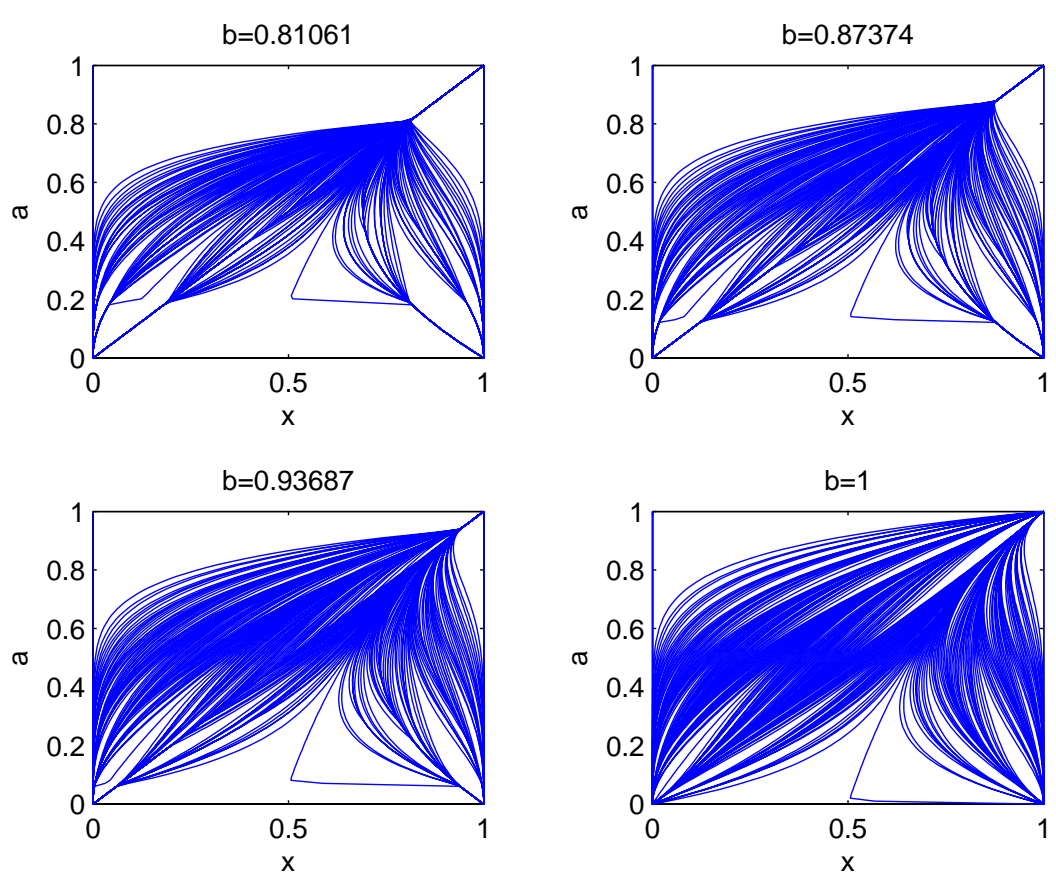

Fig. 10. Bundle plots for comparing full symmetric tent map and tent maps with different fixed $b_{i}$ while varying $a_{i}$ of the tent map with peak location $\left(a_{i}, b_{i}\right)$.

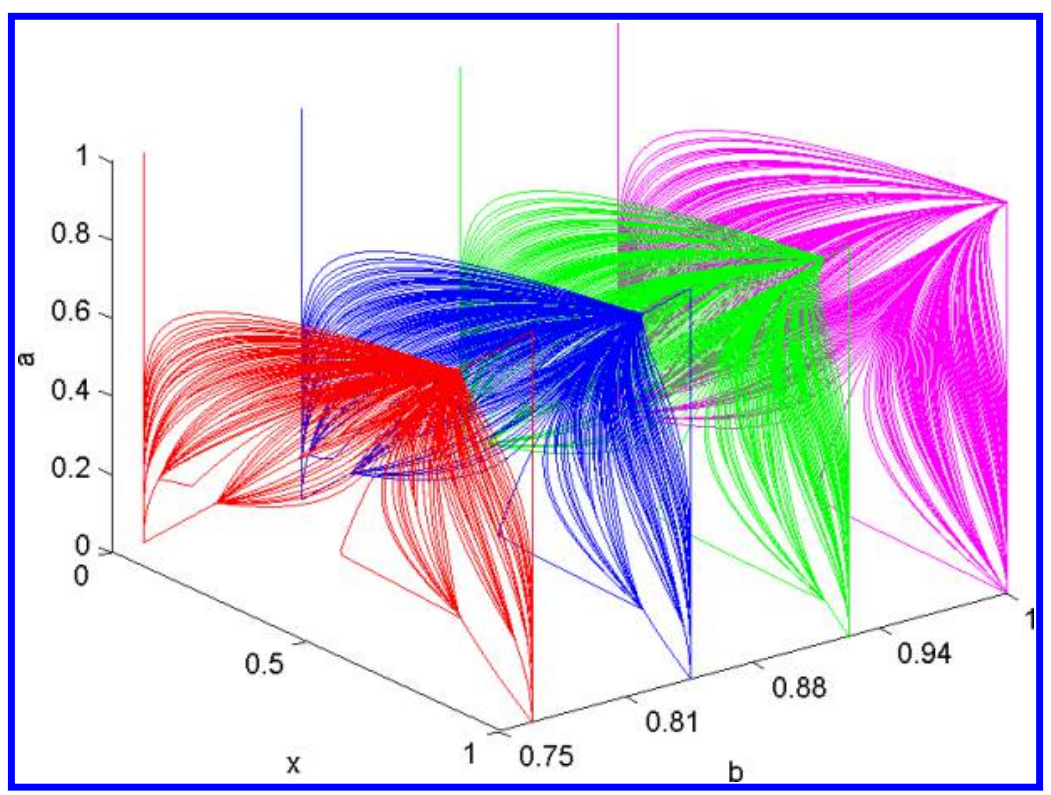

Fig. 11. 3-D bundle plots for comparing full symmetric tent map and tent maps with different fixed $b_{i}$ while varying $a_{i}$ of the tent map with peak location $\left(a_{i}, b_{i}\right)$.

fixed $b_{i}$ while varying $a_{i}$ of the tent map, with peak location $\left(a_{i}, b_{i}\right)$.

\section{Bundle Plot versus Bifurcation Plot}

The bifurcation plot keeps track of the changes of the stable fixed points. Bifurcation happens due to creation, or destruction, or stability changes of fixed points. On the other hand, we have "joint" where symbolic fibers merge (Fig. 7). As a joint exists, we lose a word/words of some length. In terms of mostly conjugacy, we say that a homeomorphic defect is born. So both bifurcation and the bundle plot seem to provide information about qualitative changes of systems, but in a different perspective. 
In order to relate and compare these two objects, we seek to answer questions like, for which parameter does joint occur? Does a joint occur when a bifurcation does? It turns out that joint happens shortly after bifurcation. In fact, the joint describes the qualitative changes of the kneading sequence as we vary a system parameter. In this section, we use the kneading theory to study the bundle's joints. $b=3.8$

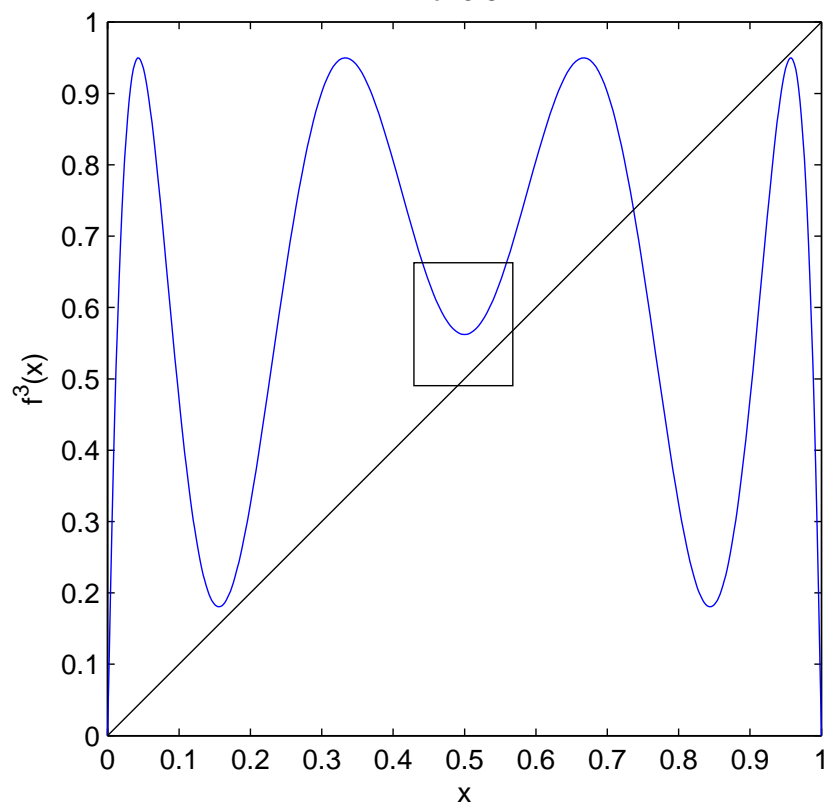

(a)

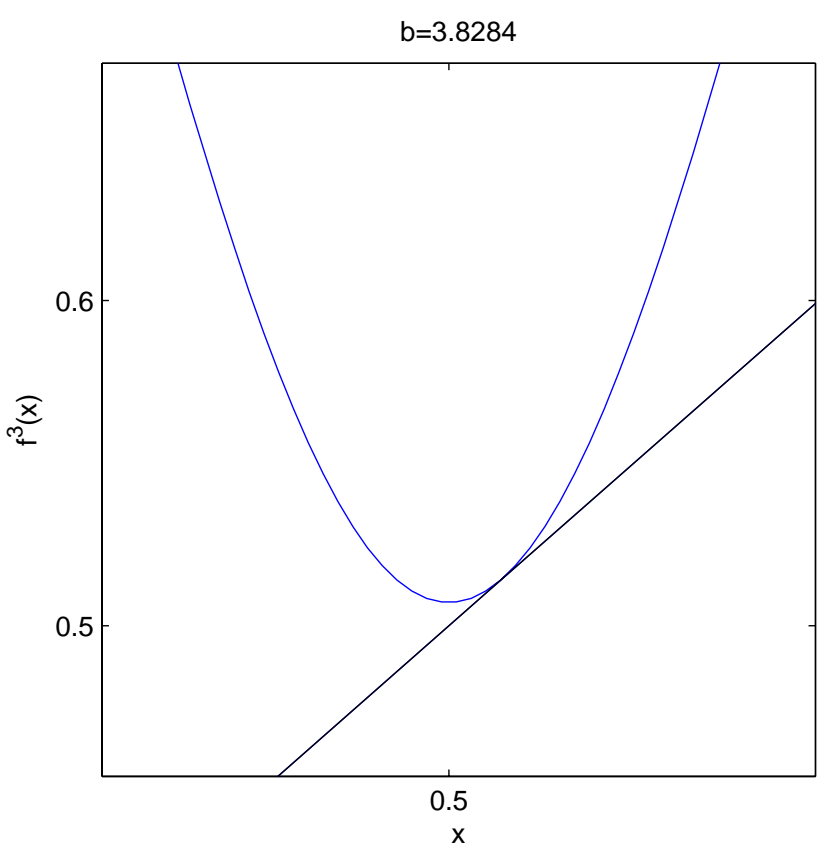

(b)

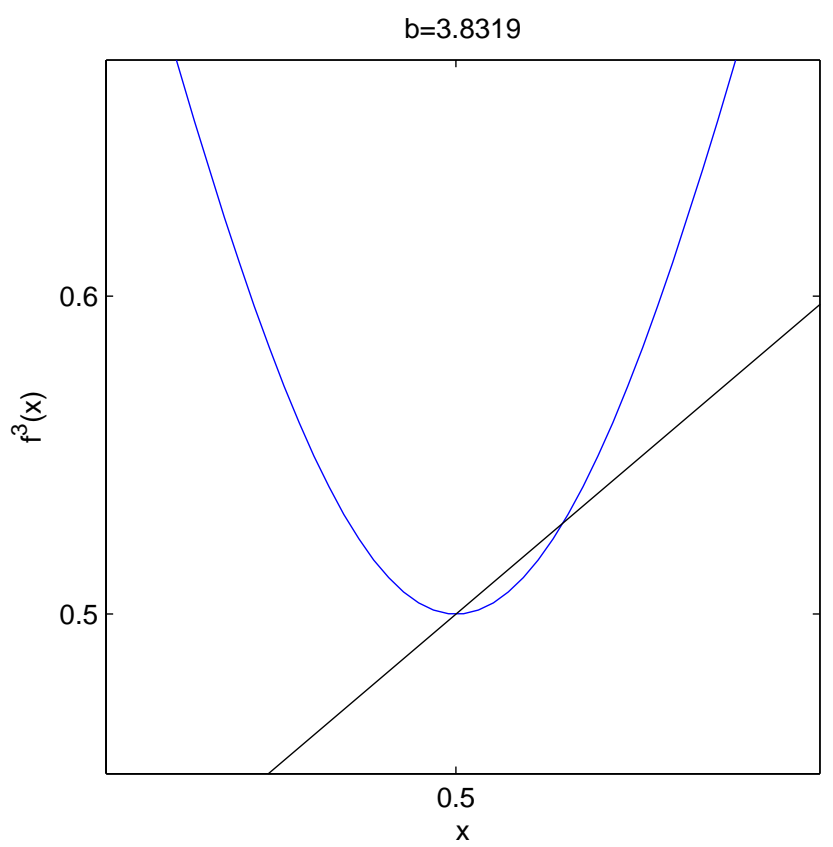

(c)

Fig. 12. Bifurcation points and Kneading points: (a) The "hump" of $f^{3}(x)$ inside the box resembles the "upside-down" of the original quadratic map; (b) As $b_{i}$ increases, the "hump" grows until it is tangent to the identity line, which indicate the opening of period-3 window; (c) As we further increase $b_{i}$ a little bit, the identity line crosses the kneading point, which implies $g_{b_{i}}^{3}(1 / 2)=1 / 2$. (a) $f^{3}(x)$, (b) bifurcation and (c) kneading intersection. 
The kneading sequence is defined as follow:

Definition 6.1 [Milnor \& Thurston, 1988]. Let $x \in$ $[0,1]$. The itinerary of $x$ under $g$ is the infinite sequence $S(x)=\left(s_{0} s_{1} s_{2} \ldots\right)$ where

$$
s_{j}= \begin{cases}0, & g^{j}(x)<\frac{1}{2}, \\ 1, & g^{j}(x)>\frac{1}{2}, \\ C, & g^{j}(x)=\frac{1}{2} .\end{cases}
$$

The kneading sequence $K(g)$ of $g$ is the itinerary of $g(1 / 2)$, i.e. $K(g)=S(g(1 / 2))$.

The kneading theory provides a critical understanding of the dynamics of unimodal maps. The kneading sequence determines which periodic orbits exist. We refer readers to Devaney's book [Devaney, 2003] and Milnor's [Milnor \& Thurston, 1988] for more details about the kneading theory, where the underlying system $g$ is a general unimodal map. Here we cite an important theorem, the Intermediate value theorem for kneading sequences [Misiurewicz, 1989], which we will use in later proof.
Theorem 3 (Intermediate value theorem for kneading sequences) [Misiurewicz, 1989]. If a oneparameter family $g_{b}$ of continuous unimodal maps depends continuously on $b$ and the topological entropy $h\left(g_{b}\right)>0$ for all $b$, then if $K\left(g_{b_{0}}\right)<K<$ $K\left(g_{b_{1}}\right)$ and $K \in \mathscr{M}$ where $\mathscr{M}$ is called the class of sequences which occur as kneading sequences of $g_{b}$ for all $b$, then there exists $b^{\prime}$ between $b_{0}$ and $b_{1}$ with $K\left(g_{b^{\prime}}\right)=K$.

Notice if we consider a full family of system $\left\{g_{b}\right\}$ where $b \in\left[b_{0}, b_{1}\right]$, the class of kneading sequences $\mathscr{M}$ would consist of all possible symbolic sequences starting with symbol $C$ between $K\left(g_{0}\right)$ and $K\left(g_{b_{1}}\right)$.

The opening of a periodic window, for instance the logistic map $x_{n+1}=g_{b_{i}}\left(x_{n}\right)=4 b_{i} x_{n}\left(1-x_{n}\right)$, can be specified by looking at the local structure of $g_{b_{i}}^{N}$. Figure 12 shows the graphs of $g_{b_{i}}^{3}=g_{b_{i}}\left(g_{b_{i}}\left(g_{b_{i}}\right)\right)$. As $b_{i}$ increases, the "hump" inside the box of Fig. 12(a), which resembles the "upside-down" of the original quadratic map, grows until it is tangent to the identity line. Then for this $b_{i}$, period- 3 window is open. As we further increase $b_{i}$ a little bit, the identity line crosses the kneading point, which implies $g_{b_{i}}^{3}(1 / 2)=1 / 2$. The kneading point becomes a point of period-3 and super-stable. In Fig. 13, we plot the opening of period- 3 with red horizontal line, and the period-3 kneading point with green horizontal line.

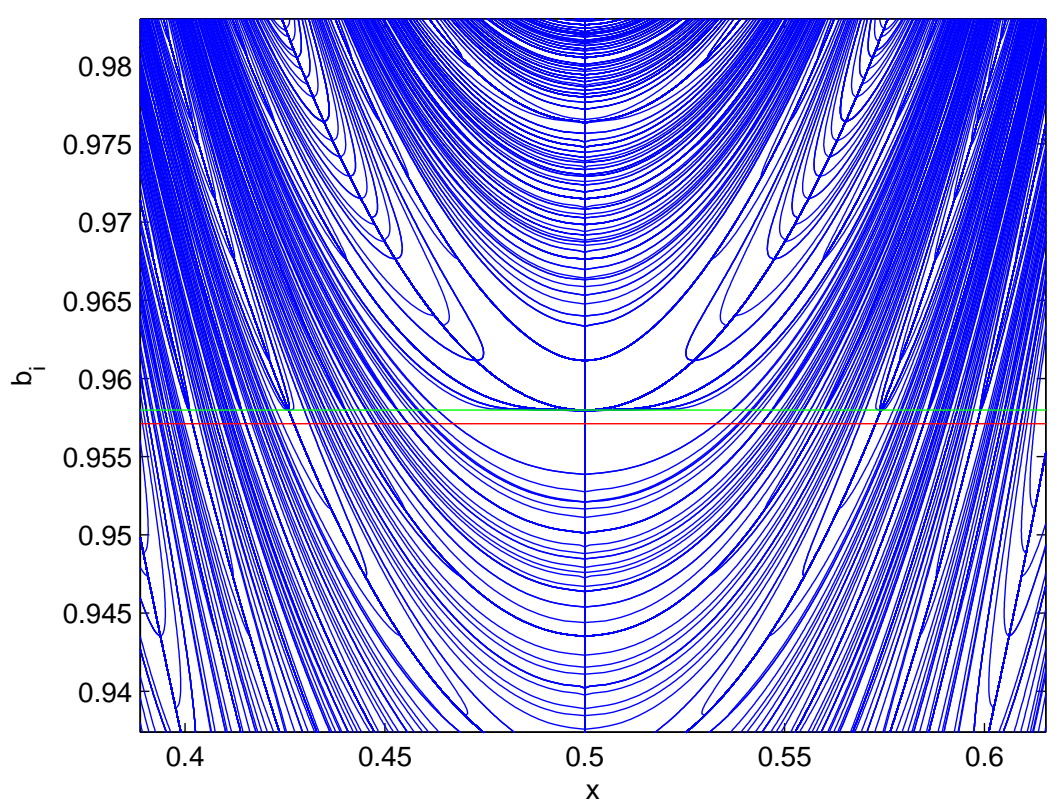

Fig. 13. The kneading point and bifurcation point: We plot the bifurcation point (the opening of period-3) with red horizontal line, and the period-3 kneading point with green horizontal line. As Theorem 4 proves, the joint (green line) happens shortly after bifurcation, and implies a period- $N$ kneading point. 


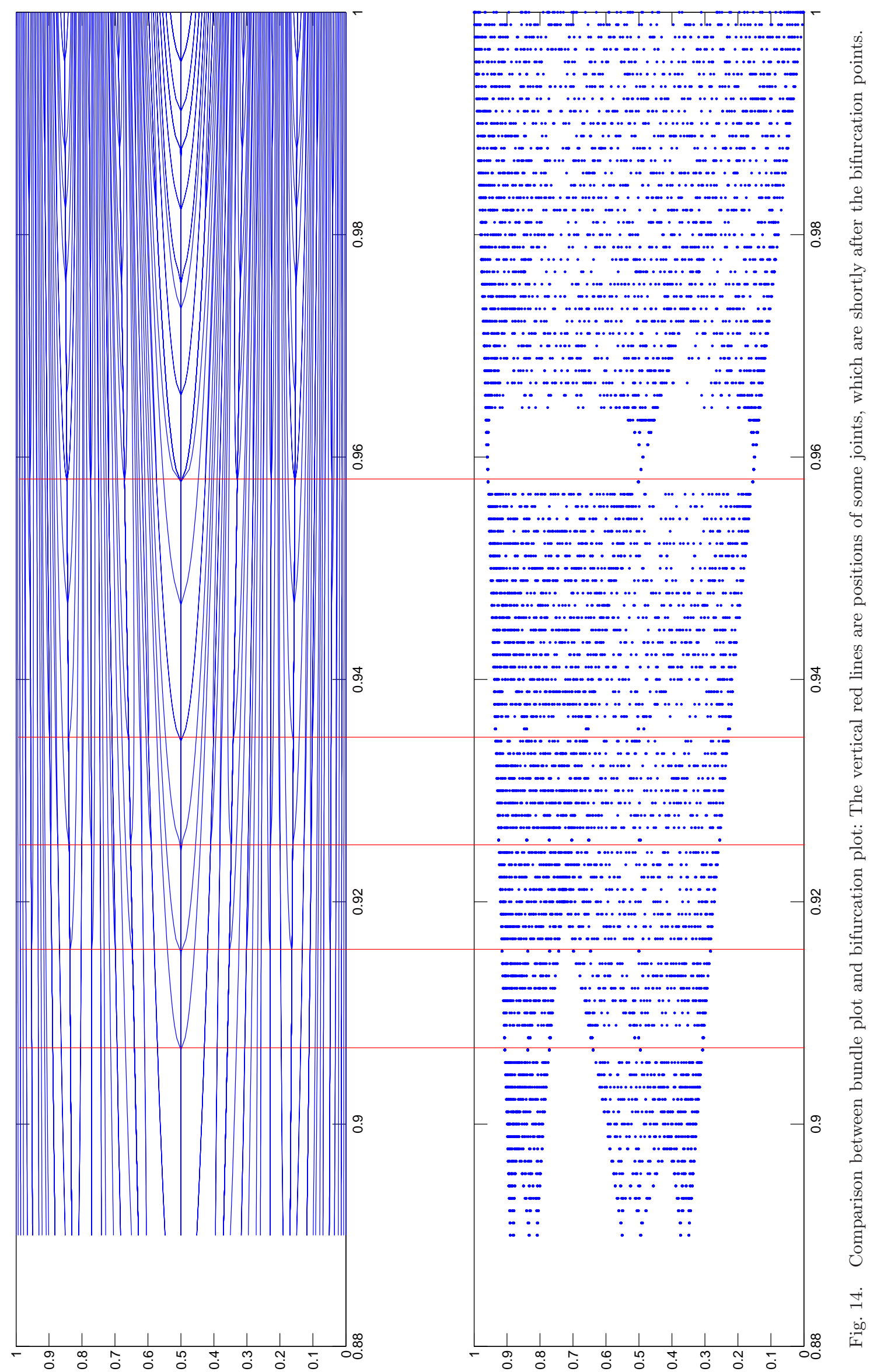


We can see that there is a joint shortly after the bifurcation point, and close to the period-3 kneading point. Is this joint at the same location as the period-3 kneading point? Or more generally, what is the relationship between the joints and the periodic kneading points? The following theorem states that the joint happens shortly after bifurcation, and implies the kneading sequence is periodic.

Theorem 4. The existence of a joint implies the kneading sequence is periodic.

Proof. Suppose we have a joint. By definition, WOLOG, assume we have $b_{\text {joint }}$ s.t. we lose a word $\omega=\underbrace{\times \times \times \cdots \times \times \times 1}_{\mathrm{N}}$ of length $N$ from the gram-

mar. Assume at $b_{\text {higher }}>b_{\text {joint }}$, we have not lost the word yet. Assume

$$
\begin{aligned}
& K\left(g_{b_{\mathrm{higher}}}\right) \\
& \quad=C * * * \cdots * * * \underbrace{\times \times \times \cdots \times \times \times 1}_{\mathrm{N}} * * * \cdots,
\end{aligned}
$$

and suppose we have lost the word $\omega=$ $\underbrace{\times \times \times \cdots \times \times \times 1}_{\mathrm{N}}$ of length $N$ at $b_{\text {lower }}<b_{\text {joint }}$, then either

$$
\begin{aligned}
& K\left(g_{b_{\text {lower }}}\right) \\
& \quad=C * * * \cdots * * * \underbrace{x \times \times \cdots \times \times \times \mathbf{C}}_{\mathrm{N}} * * * \cdots \\
& \quad=\overline{C * * * \cdots * * * \underbrace{\times \times \times \cdots \times \times \times}_{\mathrm{N}-1}}
\end{aligned}
$$

or

$$
\begin{aligned}
& K\left(g_{b_{\text {lower }}}\right) \\
& \quad=C * * * \cdots * * * \underbrace{\times \times \times \cdots \times \times \times \mathbf{0}}_{\mathrm{N}} * * * \cdots .
\end{aligned}
$$

If it is in case 1 with $K\left(g_{b_{\text {lower }}}\right)$ given by Eq. (12), then $b_{\text {joint }}=b_{\text {lower }}$ since there is no $b^{\prime}$ s.t. $K\left(g_{b_{\text {lower }}}\right)<K\left(g_{b^{\prime}}\right)<K\left(g_{b_{\text {joint }}}\right), b_{\text {lower }}<b^{\prime}<b_{\text {joint }}$ and we lose $\omega$ at $b^{\prime}$. In other words, $b_{\text {lower }}$ is the supreme of $b$ that loses $\omega$. In this case, $K\left(g_{b_{\text {joint }}}\right)$ is periodic.

If it is in case 2 with $K\left(g_{b_{\text {lower }}}\right)$ given by Eq. (13), then for

$$
\begin{aligned}
K & =C * * * \cdots * * * \underbrace{\times \times \times \cdots \times \times \times \mathbf{C}}_{\mathrm{N}} * * * \cdots \\
& =\overline{C * * * \cdots * * * \underbrace{\times \times \times \cdots \times \times \times}_{\mathrm{N}-1}}
\end{aligned}
$$

we have $K \in \mathscr{M}$ and

$$
K\left(g_{b_{\text {lower }}}\right)<K<K\left(g_{b_{\text {joint }}}\right) .
$$

Then by the Intermediate Value Theorem for Kneading Sequences (Theorem 3), there exists $b^{\prime}$ s.t. $b_{\text {lower }}<b^{\prime}<b_{\text {joint }}, K\left(g_{b^{\prime}}\right)=K$ and we lose $\omega$ at $b^{\prime}$. So in this case, $b_{\text {joint }}=b^{\prime}$ and $K\left(g_{b_{\text {joint }}}\right)$ is periodic. This completes our proof.

We give the bundle plot and the bifurcation of logistic map in Fig. 14. The vertical red lines are positions of some joints, which are shortly after the bifurcation points. On the other hand, as a wellknown phenomenon, "Period Three Implies Chaos" [Alligood et al., 1996] proves the fact that the existence of a period-3 orbit implies the existence of a large set of sensitive points. It can actually be suggested in the bundle plot. In Fig. 13, the red line indicates the period-3 opening. Later, the green line crosses a joint. Notice there are multiple fibers at this joint. As we discussed before (see Fig. 6, top right), the more symbolic fibers merge at one joint, the more words we lose at one time. Through our computation, we realize this joint is a special one in that it always has more fiber joints than others, within a given grid size of $X$ space and $\left\{b_{i}\right\}$. In other words, when the parameter $b_{i}$ is at this joint (green line), the symbolic sequences are gained/lost more than others, which says the system dynamics exhibits significantly "faster" qualitative changes at this moment.

\section{Discussion}

In this paper, we consider a different perspective of studying a system's qualitative changes due to parameter variation. We provide a bundle plot, which is from commuters, to picture such evolution of the symbolic space. The joint implies qualitative changes where the kneading points become periodic. And it happens shortly after bifurcation.

The construction of bundle plots is applicable to other unimodal systems. Generally speaking, we only require the family of maps $\left\{g_{b_{i}}\right\}$ piecewise invertible and

- $\hat{g}_{b_{i}}^{-1}$ is piecewise continuous on $Y$; 
- $\hat{g}_{b_{i}}^{-1}$ is piecewise Lipshitz continuous, with Lipshitz continuous $L<1$,

which guarantees there is a unique commuter. We believe that the family maps $g_{b_{i}}$ can be generalized to be maps with positive Lyapunov exponents, since such "average" contraction of $g_{b_{i}}^{-1}$ can also result in a unique commuter, which gives rise to the bundle plot. On the other hand, we are also trying to extend this method to higher dimensional systems, where we assume that the dynamical systems under consideration are presented to us with a known symbolic dynamic partitioning.

We also note that the study in this paper is based on a given system. In fact, in the construction of bundle, the base function $g_{b_{1}}$ can be given by a time series data. In this case, we provide a family of system $\left\{g_{b_{i}}\right\}$, and see how these systems match the original data set.

\section{References}

Alligood, K. T., Sauer, T. D. \& Yorke, J. A. [1996] Chaos: An Introduction to Dynamical Systems, 3rd edition (Springer).

-Bollt, E. M. \& Skufca, J. D. [2010] "Comparing dynamical systems by defective conjugacy: A symbolic dynamics interpretation of commuter functions," Physica D 239, 579-590.
Devaney, R. L. [2003] An Introduction to Chaotic Dynamical Systems, 2nd edition (Westview Press).

Kantz, H. \& Schreiber, T. [2003] Nonlinear Time Series Analysis, 2nd edition (Cambridge University Press, USA).

- Milnor, J. \& Thurston, W. [1988] "On iterated maps of the interval dynamical systems," Lecture Notes in Math. 1342, 465-563.

-Misiurewicz, M. [1989] "Jumps of entropy in one dimension," Fund. Math. 132, 215-216.

Ott, E. [2002] Chaos in Dynamical Systems, 2nd edition (Cambridge University Press, USA).

- Poincaré, H. \& Magini, R. [1899] "Les méthodes nouvelles de la Mécanique céleste," Il Nuovo Cimento Ser. 10, 128-130.

Rowland, T. "Fiber Bundle," A Wolfram Web Resource, created by Eric W. Weisstein. http://mathworld. wolfram.com/FiberBundle.html.

-Skufca, J. D. \& Bollt, E. M. [2008] "A concept of homeomorphic defect for defining mostly conjugate dynamical systems," Chaos 18, 013118.

Wheeden, R. L. \& Zygumund, A. [1977] Measure and Integral, an Introduction to Real Analysis (Marcel Dekker, Inc.).

Zheng, J., Bollt, E. M. \& Skufca, J. D. [2011] "Regularity of commuter functions for homeomorphic defect measure in dynamical systems model comparison," DCDIS Ser. A: Math. Anal. 18, 363-382.

Zheng, J., Bollt, E. M. \& Skufca, J. D. [2012] "Comparing dynamical systems by graph matching method," submitted to Physica D. 